\title{
外源多胺对盆栽花生盐胁迫的缓解作用
}

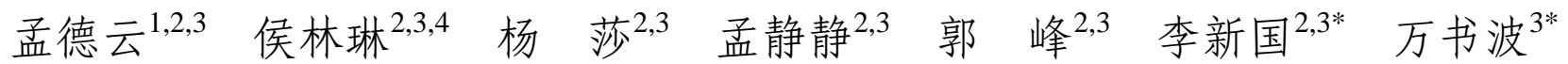 \\ ${ }^{1}$ 山东大学生命科学学院, 济南 $250100 ;{ }^{2}$ 山东省农业科学院生物技术研究中心, 济南 $250100 ;{ }^{3}$ 山东省作物遗传改良与生态生理重点实验室, 济南 \\ $250100 ;{ }^{4}$ 青岛农业大学农学与植物保护学院, 青岛 266109
}

摘 要 为探讨外源多胺对花生(Arachis hypogaea)抗盐性的影响, 以盆栽花生 “花育 22’为试验材料, 通过叶面喷施1 $\mathrm{mmol} \cdot \mathrm{L}^{-1}$ 腐胺(Put)、 $1 \mathrm{mmol} \cdot \mathrm{L}^{-1}$ 亚精胺 $(\mathrm{Spd}) 、 1 \mathrm{mmol} \cdot \mathrm{L}^{-1}$ 精胺 $(\mathrm{Spm})$ 的方法, 研究多胺对 $150 \mathrm{mmol} \cdot \mathrm{L}^{-1} \mathrm{NaCl}$ 胁迫下盆栽花生 的缓解作用。结果表明, 与对照 $(\mathrm{CK})$ 相比, 盐胁迫显著抑制了花生植株的生长与英果产量, 降低了叶绿素含量和抗氧化酶活 性, 丙二醛(MDA)含量、叶片相对电导率增加; 在盐胁迫下, 叶面喷施Put、Spd、Spm处理均可有效促进花生植株的生长, 提 高了超氧化物歧化酶(SOD)、过氧化物酶(POD)、过氧化氢酶(CAT)的活性, 增强植株抗氧化能力, 显著降低了花生叶片相对 电导率和MDA的积累量, 从而缓解盐胁迫对质膜的过氧化伤害; 提高了叶绿素含量, 促进了植株高度的生长与分支数增多, 增加了干物质积累量, 从而提高了花生荚果产量; 其中, Spm处理引起的变化幅度大于Spd和Put处理。研究结果说明, 多胺有 利于花生幼苗在盐胁迫下活性氧代谢和光合色素含量的提高, 促进花生植株的生长, 降低盐胁迫对花生植株的抑制作用, 且 Spm处理的效果最好。

关键词 活性氧清除酶, 花生, 多胺, 盐胁迫, 产量

引用格式: 孟德云, 侯林琳, 杨莎, 孟静静, 郭峰, 李新国, 万书波 (2015). 外源多胺对盆栽花生盐胁迫的缓解作用. 植物生态学报, 39, 1209-1215. doi: $10.17521 /$ cjpe.2015.0117

\section{Exogenous polyamines alleviating salt stress on peanuts (Arachis hypogaea) grown in pots}

MENG De-Yun ${ }^{1,2,3}$, HOU Lin-Lin ${ }^{2,3,4}$, YANG Sha ${ }^{2,3}$, MENG Jing-Jing ${ }^{2,3}$, GUO Feng ${ }^{2,3}$, LI Xin-Guo ${ }^{2,3^{*}}$, and WAN Shu-Bo ${ }^{3 *}$

${ }^{1}$ School of Life Science of Shandong University, Jinan 250100, China; ${ }^{2}$ Biotechnology Research Center, Shandong Academy of Agricultural Sciences, Jinan 250100, China; ${ }^{3}$ Shandong Provincial Key Laboratory of Crop Genetic Improvement, Ecology and Physiology, Jinan 250100, China; and ${ }^{4}$ College of Agronomy and Plant Protection, Qingdao Agricultural University, Qingdao 266109, China

\section{Abstract}

Aims Soil salinity is a major limiting factor for plant establishment, development and productivity. In recent years, the contradiction between oil crops and food crops for land is increasingly prominent. In order not to take up the land for food, peanut planting on saline-alkali land could be a promising option. However, peanuts have been rarely grown in saline-alkali land, which may be due to the reduction of peanut yield caused by salt stress. Therefore, research of peanut salt resistance has important practical significance.

Methods In order to investigate the effects of exogenous polyamines on peanut (Arachis hypogaea) grown in pots under salt stress, 'Huayu 22', one of the peanut cultivars, was used as materials by being foliar-sprayed with $1 \mathrm{mmol} \cdot \mathrm{L}^{-1}$ putrescine (Put), $1 \mathrm{mmol} \cdot \mathrm{L}^{-1}$ spermidine (Spd) and $1 \mathrm{mmol} \cdot \mathrm{L}^{-1}$ spermine (Spm) to elucidate the role of exogenous polyamines on peanuts under $150 \mathrm{mmol} \cdot \mathrm{L}^{-1} \mathrm{NaCl}$.

Important findings Results showed that growth, yield, chlorophyll contents and antioxidant enzyme activities of peanut seedling decreased, however, malondialdehyde (MDA) content and relative electrolytic leakage increased under salt stress. Meanwhile, exogenous polyamines significantly improved the activities of superoxide dismutase (SOD), peroxidase (POD) and catalase (CAT), and reduced the relative electrolytic leakage and MDA content in peanut leaves under salt stress and thus alleviating the oxidative damage of salt stress on plasma membrane. It is obvious that exogenous polyamines could improve chlorophyll contents, plant height, number of branch and the amount of dry matter accumulation, even pod yield under salt stress. Among these three polyamines, the effects of exogenous Spm on alleviating salt stress were most effective These results showed that exogenous polyamines, especially Spm, were favorable for the seedlings to

收稿日期Received: 2015-04-17 接受日期Accepted: 2015-11-17

* 共同通讯作者Co-author for correspondence (E-mail: lixinguo@tom.com; wansb@saas.ac.cn) 
increase reactive oxygen metabolism and photosynthesis, which improved peanut growth and reduced the inhibitory effects of salt stress on peanuts.

Key words antioxidant enzyme, peanut, polyamines, salt stress, yield

Citation: Meng DY, Hou LL, Yang S, Meng JJ, Guo F, Li XG, Wan SB (2015). Exogenous polyamines alleviating salt stress on peanuts (Arachis hypogaea) grown in pots. Chinese Journal of Plant Ecology, 39, 1209-1215. doi: 10.17521/cjpe.2015.0117

土壤盐渍化是影响农业生产和生态环境的严重 问题, 据统计, 全球有 $20 \%$ 的耕地受到不同程度的 盐渍化(李建国等, 2012), 严重影响作物生长, 降低 作物产量。花生(Arachis hypogaea) 是我国重要的油 料经济作物, 具有重要的营养价值和经济价值。近 年来油料作物与粮食作物争地的矛盾日益突出, 为 了不与粮争地, 将花生种植在盐碱地上是不错的选 择(万书波, 2009)。但是在中国, 花生很少种植在盐 碱地, 这可能与盐胁迫影响花生产量有关。因此, 提 高花生耐盐性研究具有重要的现实意义。

多胺是一类广泛存在于动植物体内具有强烈的 生物活性的多聚阳离子、低分子量的脂肪族含氮碱。 植物细胞中的多胺主要包括腐胺(Put)、亚精胺(Spd) 和精胺(Spm)。研究发现多胺与植物体的生长、发育 以及对环境胁迫的响应等多种生理过程相关, 并且 参与植物体中的信号转导 (Galston \& Sauheny, 1990)。研究发现多胺可以通过多种机制响应非生物 胁迫, 如影响清除活性氧的酶系统(徐仰仓等, 2001)、非酶系统(Maccarrone et al., 1998)以及作为 第二信使参与植物的逆境胁迫信号传递(Liu et al., 2000; Sudha \& Ravishankar, 2003)等。

研究表明, 盐胁迫下植物体内源多胺含量发生 剧烈变化, 且因植物种类不同、组织部位、胁迫时 间的不同而表现出差异(Liu et al., 2007)。外施多胺 可提高植物耐盐性, 但不同植物种类及不同种类多 胺的施用效应差异较大。例如, 外源Put和Spd对减 轻盐敏感型水稻(Oryza sativa)所受的胁迫伤害有一 定的作用, 而施用外源Spm却没有任何明显效果 (Ndayiragije \& Lutts, 2007)。施用外源Spm能够通过 调节叶绿体抗氧化系统显著缓解黄瓜(Cucumis sativus)幼苗受到的盐胁迫损伤(Shu et al., 2013)。在花 生中, 关于多胺的研究甚少, 只有极少数关于多胺 氧化酶及衰老的研究, 缺乏对多胺在花生抗逆性方 面的研究。本文以“花育22, 为试验材料, 重点研究盐 胁迫下, 喷施外源多胺Put、Spd、Spm对盆栽花生植 株生长及产量的影响, 以期为盐碱地花生种植提供
理论支持。

\section{1 材料和方法}

\section{1 材料与处理}

试验于山东省农业科学院饮马泉试验农场进 行, 供试材料为花生“花育22’ (种子由山东省农业科 学院提供)。土壤为该农场 $0-20 \mathrm{~cm}$ 土层土壤, 所用 栽培盆为内径 $30 \mathrm{~cm}$ 、高 $40 \mathrm{~cm}$ 的瓷花盆。试验于5 月16日播种, 每盆三穴, 每穴两粒, 出苗后每穴保 留 1 株健壮苗。于 6 月 3 日与 7 月 3 日两次浇 150 $\mathrm{mmol} \cdot \mathrm{L}^{-1} \mathrm{NaCl}$ 溶液, 每盆用 $2 \mathrm{~L}$ 浇透(土壤最终盐浓 度为 $0.2 \%)$, 以浇纯净水处理为对照 (CK)。于始花 期、下针期、结荚期分别叶面喷施Put、Spd、Spm 溶液, 浓度均为 $1 \mathrm{mmol} \cdot \mathrm{L}^{-1}$, 每盆喷施 $30 \mathrm{~mL}$ (刘俊 等, 2006; Roychoudhury et al., 2011; 王晓云等, 1999), CK与单纯盐处理叶面喷施等量纯净水, 共3 次喷施处理, 均在无风傍晚进行。处理7天后取样测 定相关指标, 每个处理5个重复。

\section{2 取样及指标测定}

分别于7月14日(始花期, 记为FE)、7月29日(下 针期，记为FP)、8月20日(结荚期，记为PP)、9月 26 日(收获期, 记为PF)取样测定相关指标。每次选取 5 株完整植株, 根部深入土壤 $30 \mathrm{~cm}$ 进行挖掘。将总叶 片、枝茎、根系和荚果样品分开置于 $105{ }^{\circ} \mathrm{C}$ 的烘箱 内杀青 $30 \mathrm{~min}, 85{ }^{\circ} \mathrm{C}$ 烘干至恒质量后称量; 收获期 分别进行植株高度、侧枝数、第一对侧枝平均长度 的统计与测定; 各时期取功能叶倒三叶鲜样测定叶 绿素含量、相对电导率, 液氮速冻倒三叶用于测定 活性氧清除酶活性和丙二醛(MDA)含量。

乙醇浸提法测定叶绿素含量(李合生, 2000); 相 对电导率用DDS-11A 型电导率仪(上海大普仪器有 限公司, 上海)测定; MDA含量采用硫代巴比妥酸 (TBA)法测定(赵世杰等, 1994); 超氧化物歧化酶 (SOD) 活性采用氮蓝四唑法测定 (Giannopolitis \& Ries, 1977); 过氧化物酶(POD)活性采用愈创木酚 法测定(曾韶西等, 1997); 过氧化氢酶(CAT)活性采 
用过氧化氢紫外线法测定(张治安和陈展宇, 2008)。 每个指标重复测定5次, 取平均值。

\section{3 数据处理}

采用SigmaPlot 10.0进行数据处理和绘图, SPSS 16.0进行显著性分析。

\section{2 实验结果}

\section{1 盐胁迫下外源多胺对花生植株农艺性状的影响}

由表1可以看出, 盐胁迫显著抑制了花生的主 茎高、侧枝长和分枝数, 与CK相比, 主茎高度降低 了 $15.42 \%$, 侧枝长度下降了 $16.07 \%$, 分枝数减少了 $32.52 \%$ 。外施多胺可明显改善花生营养生长, 部分 缓解盐胁迫造成的抑制作用, 花生主茎高、侧枝长、 分枝数均较单一盐胁迫显著增加。其中Spm的施用 对花生主茎高和侧枝长影响最显著, 较单一盐胁迫 分别增加了 $11.96 \%$ 和 $16.67 \%$; Put和Spm对分枝数的 增加幅度相同, 均比单一盐胁迫植株增加了 $20.68 \%$, Spd 对分枝数的增加最显著, 增加达 $31.02 \%$ 。可见, 外源多胺能有效地缓解盐胁迫对花 生生长的抑制作用。

\section{2 盐胁迫下外源多胺对花生植株生物量的影响}

盐胁迫降低了各时期花生总生物量的积累(图 1), 较 CK 的 FE、FP、 PP 和 PF 时期分别下降了 $22.86 \% 、 36.58 \% 、 38.22 \%$ 和 $25.54 \%$ 。外源多胺能提 高花生植株总生物量, 其中Spm对总生物量的贡献 大于Spd和Put处理, 各时期内相对于单纯盐胁迫处 理分别增加了 $19.15 \% 、 30.96 \% 、 47.48 \%$ 和 $18.99 \%$, 其中在FP和PP两时期的增加幅度尤为明显。

\section{3 盐胁迫下外源多胺对花生叶绿素含量的影响}

由图2可知, 花生叶片叶绿素含量在结荚期达 到峰值, 随后逐渐进入衰老进程, 叶绿素含量下降 迅速。与CK相比, 盐胁迫降低了各时期的叶绿素含 量。叶面喷施外源多胺能够减缓叶绿素含量的下降。 其中Spm效果最明显, 在FE、FP、PP和PF等时期叶 绿素含量分别比单纯盐胁迫处理高出 $13.45 \%$ 、 14.38\%、19.95\%、23.58\%。外源Put和Spd两处理也 能提高叶绿素含量, 但两个处理间差异不明显。

\section{4 盐胁迫下外源多胺对花生叶片活性氧清除酶 活性的影响}

在整个生育时期内花生叶片活性氧清除酶SOD、 POD、CAT活性整体呈先增加后下降的趋势。盐胁 迫处理后各时期SOD、POD、CAT活性都明显低于
表1 盐胁迫下外源多胺对花生植株农艺性状的影响(平均值土标准偏差) Table 1 Effects of exogenous polyamines on agronomic characters of Arachis hypogaea seedlings under salt stress (mean $\pm S D$ )

\begin{tabular}{lccc}
\hline $\begin{array}{l}\text { 处理 } \\
\text { Treatment }\end{array}$ & $\begin{array}{c}\text { 主茎高 } \\
\text { Plant height }(\mathrm{cm})\end{array}$ & $\begin{array}{c}\text { 侧枝长 } \\
\text { Branch length }(\mathrm{cm})\end{array}$ & $\begin{array}{c}\text { 分枝数 } \\
\text { Branch number }\end{array}$ \\
\hline $\mathrm{CK}$ & $31.33 \pm 0.63^{\mathrm{a}}$ & $32.17 \pm 0.31^{\mathrm{a}}$ & $14.33 \pm 0.24^{\mathrm{a}}$ \\
$\mathrm{NaCl}$ & $26.50 \pm 0.25^{\mathrm{c}}$ & $27.00 \pm 0.48^{\mathrm{b}}$ & $9.67 \pm 0.24^{\mathrm{c}}$ \\
$\mathrm{NaCl}+\mathrm{Put}$ & $27.67 \pm 0.29^{\mathrm{bc}}$ & $30.50 \pm 0.85^{\mathrm{ab}}$ & $11.67 \pm 0.47^{\mathrm{b}}$ \\
$\mathrm{NaCl}+\mathrm{Spd}$ & $29.33 \pm 0.52^{\mathrm{b}}$ & $29.25 \pm 0.64^{\mathrm{ab}}$ & $12.67 \pm 0.24^{\mathrm{ab}}$ \\
$\mathrm{NaCl}+\mathrm{Spm}$ & $29.67 \pm 0.58^{\mathrm{b}}$ & $31.50 \pm 0.38^{\mathrm{a}}$ & $11.67 \pm 0.24^{\mathrm{b}}$ \\
\hline
\end{tabular}

Put, 腐胺; Spd, 亚精胺; Spm, 精胺。同一列不同小写字母表示处理间差 异显著 $(p<0.05)$ 。

Put, putrescine; Spd, spermidine; Spm, spermine. Different letters indicate significant differences among treatments $(p<0.05)$.

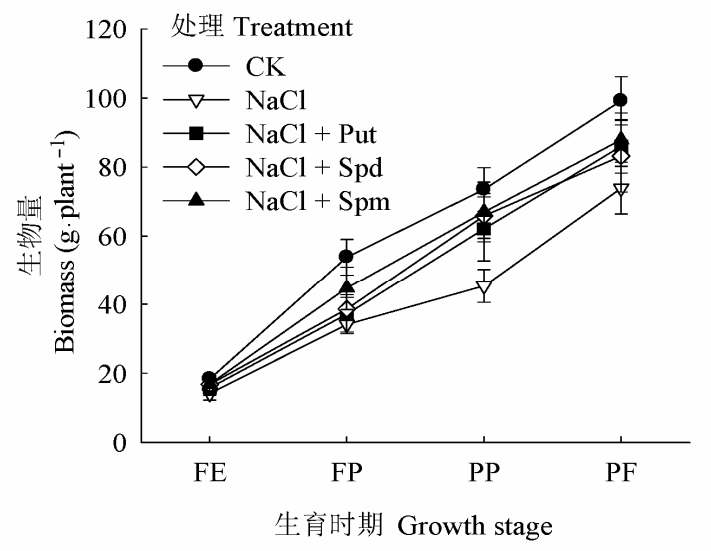

图1 盐胁迫下外源多胺对花生植株总生物量的影响(平均 值土标准偏差)。FE、FP、PP和PF分别代表始花期、下针期、 结荚期和收获期。Put、Spd、Spm同表1。

Fig. 1 Effects of exogenous polyamines on total biomass of Arachis hypogaea under salt stress (mean $\pm S D$ ). FE, FP, PP and $\mathrm{PF}$ represent flower early stage, flower-pegging stage, pod-setting stage and pod filling stage, respectively. Put, Spd, Spm see Table 1.

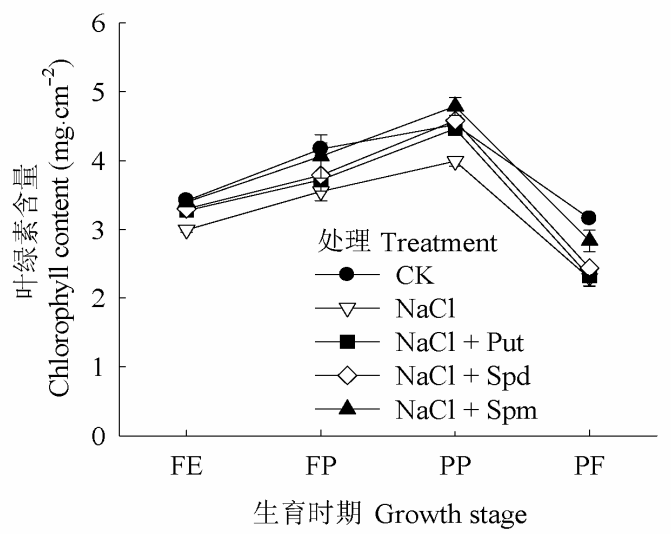

图2 盐胁迫下外源多胺对花生叶片叶绿素含量的影响(平 均值土标准偏差)。FE、FP、PP和PF分别代表始花期、下针 期、结荚期和收获期。Put、Spd、Spm同表1。

Fig. 2 Effects of exogenous polyamines on leaf chlorophyll content of Arachis hypogaea under salt stress (mean $\pm S D$ ). FE, FP, PP and PF represent flower early stage, flower-pegging stage, pod-setting stage and pod filling stage, respectively. Put, Spd, Spm see Table 1. 
CK，外源喷施多胺均提高了活性氧清除酶活性(图 3)。其中以喷施Spm缓解效果最好，与单纯盐胁迫相 比, FE、FP、PP和PF 4 个时期的SOD活性分别增加 了 $19.20 \% 、 20.87 \% 、 37.35 \%$ 和 $56.73 \%$, POD活性分 别增加了 $18.05 \%$ 、33.46、25.66\%和44.63\%, CAT活 性分别增加了 $47.67 \% 、 45.22 \% 、 29.49 \%$ 和 $52.52 \%$ 。 喷施Put处理和Spd处理对活性氧清除酶活性的影响
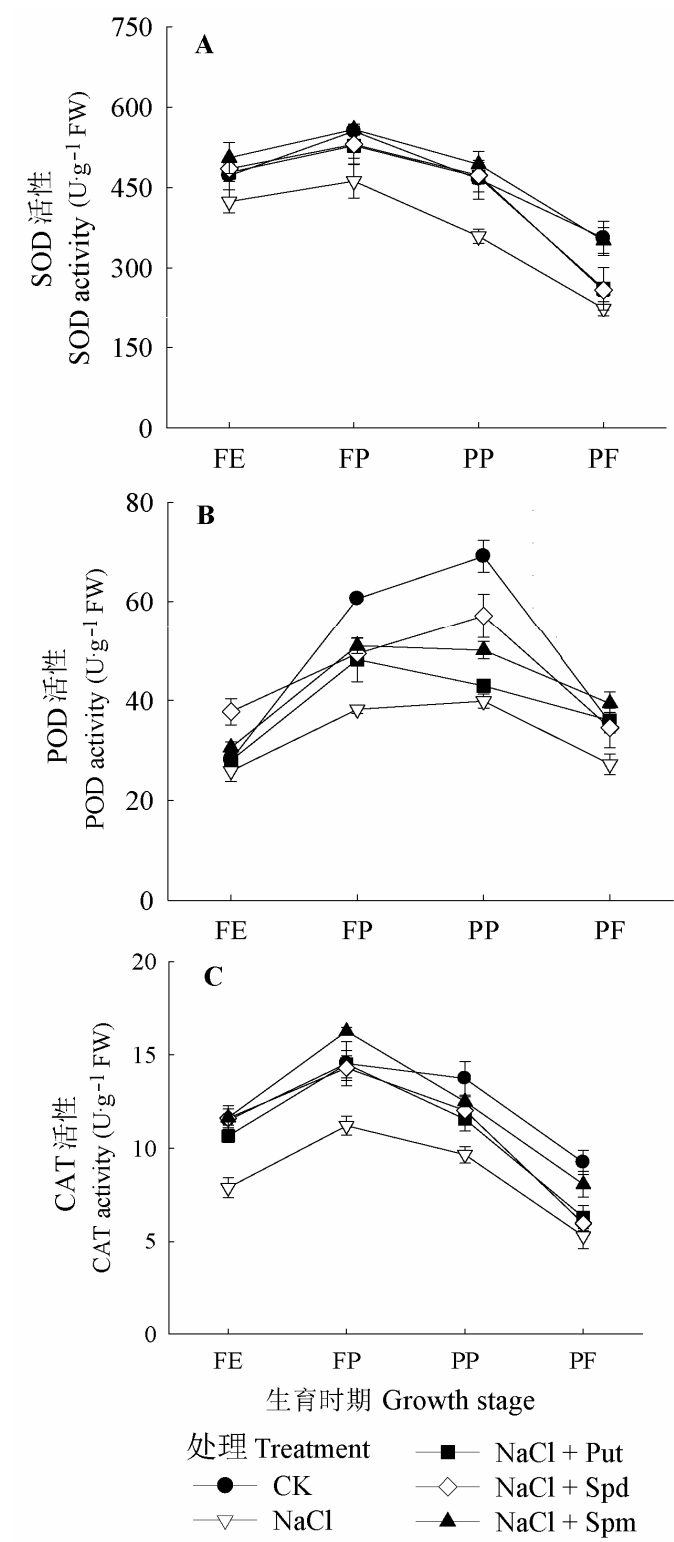

图3 盐胁迫下外源多胺对花生叶片超氧化物歧化酶(SOD) 活性(A)、过氧化物酶(POD)活性(B)、过氧化氢(酶(CAT)活性 (C)的影响(平均值土标准偏差)。FE、FP、PP和PF分别代表始 花期、下针期、结荚期和收获期。Put、Spd、Spm同表1。

Fig. 3 Effects of exogenous polyamines on superoxide dismutase (SOD) activity (A), peroxidase (POD) activity (B) and catalse (CAT) activity (C) of Arachis hypogaea leaves under salt stress (mean $\pm S D$ ). FE, FP, PP and PF represent flower early stage, flower-pegging stage, pod-setting stage and pod filling stage, respectively. Put, Spd, Spm see Table 1.
整体上小于 Spm处理，其中Put和Spd两处理对SOD 活性的影响没有明显差异, Spd处理对POD活性和 CAT活性的影响略强于Put处理。说明施外源多胺可 以提高花生叶片活性氧清除酶活性，有利于对活性 氧的清除, 缓解盐胁迫下因活性氧积累对花生植株 造成的伤害, 且Spm和Spd处理的作用效果好于Put。

\section{5 盐胁迫下外源多胺对花生叶片生物膜完整性 的影响}

植物细胞受胁迫损伤程度可通过细胞膜相对电 导率和MDA含量的变化来反映。随着植株的生长发 育, 花生叶片细胞膜相对电导率和MDA含量都逐 渐增加。由图4可知, 与CK相比, 盐胁迫增大了生物 膜损伤程度，各生育时期的相对电导率和MDA含 量均高于对照。外源喷施多胺可有效地降低盐胁迫 对花生生物膜的损伤。喷施Put、Spd、Spm与单纯 盐胁迫相比，相对电导率在FE期分别下降了 $9.98 \%$ 、
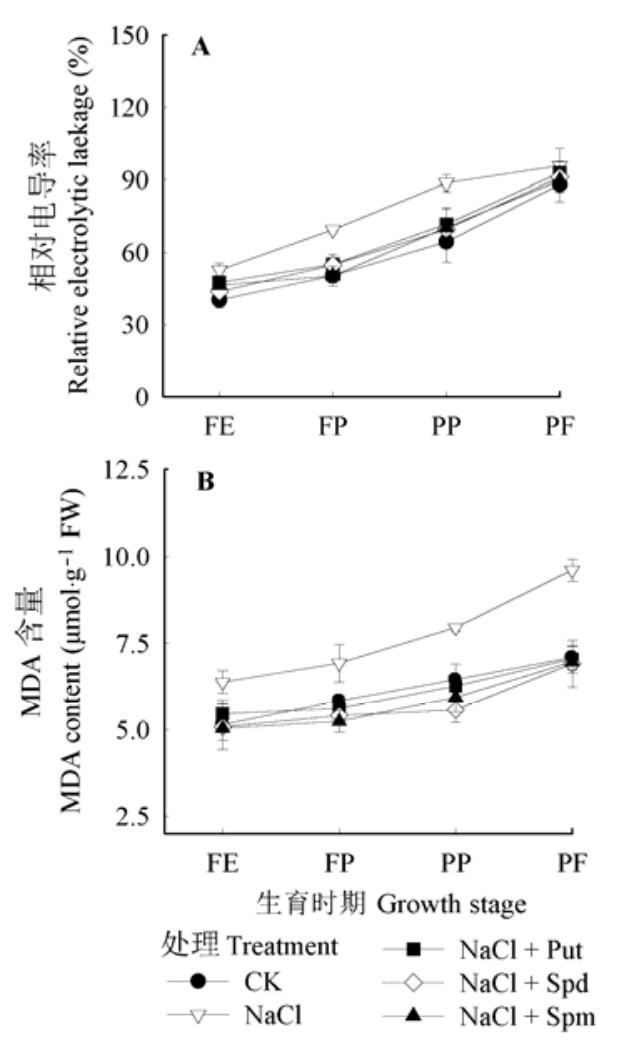

图4 盐胁迫下外源多胺对花生叶片相对电导率(A)和丙二 醛(MDA)含量(B)的影响(平均值土标准偏差)。FE、FP、PP和 PF分别代表始花期、下针期、结荚期和收获期。Put、Spd、 Spm同表1。

Fig. 4 Effects of exogenous polyamines on relative electrolytic leakage (A) and malondialdehyde (MDA) content (B) of Arachis hypogaea leaves under salt stress (mean $\pm S D$ ). FE, FP, $\mathrm{PP}$ and $\mathrm{PF}$ represent flower early stage, flower-pegging stage, pod-setting stage and pod filling stage, respectively. Put, Spd, Spm see Table 1. 
$17.08 \%$ 和 $11.80 \%$, 在FP期分别增加下降了 $20.80 \%$ 、 $21.15 \%$ 和 $27.63 \%$, 而在PP期与 PF期，3种处理之间没 有明显差异。喷施Put、Spd、Spm与单纯盐胁迫相比, MDA 含量在FE期分别下降了 $14.41 \%$ 、20.34\%和 $21.02 \%$, 在 FP 期分别下降了 $18.93 \%$ 、 $21.95 \%$ 和 $24.34 \%$, 在 PP 期分别下降了 $21.26 \%$ 、29.95\% 和 $25.42 \%$, 在 PF期 3 种处理之间没有明显差异。由此可 见, 盐胁迫使花生叶片生物膜透性增大, 可能与活性 氧过量积累引起的膜脂过氧化程度加剧有关, 外源喷 施多胺, 尤其是Spd和Spm, 能有效地降低叶片相对电 导率和MDA含量, 减轻盐胁迫对花生植株的伤害。

\section{6 盐胁迫下外源多胺对花生产量的影响}

从表2可以看出, 盐胁迫显著降低了花生单株 果针数、单株总果数和饱果数, 从而降低了单株产 量, 外源喷施多胺能够在一定程度上提高盐胁迫下 的花生产量, Put、Spd、Spm处理相对于单纯盐胁迫 产量分别提高了 $10.34 \%$ 、13.38\%和 $16.90 \%$ 。3种多 胺对果针数及饱果数均有明显影响, 其中Spm的作 用最显著, 从而能够显著提高单株产量。

\section{3 讨论}

盐胁迫主要通过抑制或诱导多种酶系统以及气 孔导度等途径来降低光合作用和能量代谢, 抑制植 物组织的生长与发育, 从而降低植物干物质积累与 植物产量(Parida \& Das, 2005)。用Put和Spd浸种均可 缓解盐胁迫对大麦(Hordeum vulgare)幼苗的盐害, 促进生长和干物质积累(Sun et al., 2002)。Krishnamurthy和Bhagwat (1989)的研究表明, 高浓度 $\mathrm{NaCl}$ 胁 迫下, 水稻种子萌发和生长受阻, 但外源Put可恢复 种子萌发和生长, 促进盐胁迫下水稻禾苗的生长, 增加稻谷产量。

本实验中叶面喷施外源多胺能够缓解盐胁迫对 植株生长的抑制, 提高花生主茎高度和侧枝长度的 生长, 分枝数增多(表1), 干物质积累量增加(图1),
荚果产量提高(表2)。

光合作用为植物生长发育提供物质与能量, 是 植物生长发育的基础。而叶绿素是光合作用中光反 应阶段色素蛋白复合体的重要组成部分, 叶绿素含 量的高低直接影响植物的光合作用能力 (Maslenkova et al., 1993)。盐胁迫不仅能提高叶绿素 酶的活性, 加速叶绿素的降解, 也能抑制叶绿素的 合成( フ丰秋等, 1997)。本实验中盐胁迫处理显著降 低了叶绿素含量, 喷施3种多胺在生育前期能够有 效地提高叶绿素含量的积累, 在生育后期能有效地 缓解叶绿素的降解速率(图2)。有研究表明, 外源多 胺能够延缓黑暗诱导下离体叶片的衰老, 特别是 Spd和Spm具有明显的保绿和抑制蛋白质降解的作 用(蒋琳等, 1993), 王晓云等(1999)也发现大田抗衰 老能力强的花生品种连体叶片内具有较高的Spd和 Spm含量, 从而可减缓叶绿素的降解。

胁迫条件下叶绿素的降解与活性氧有关。Shioi 等(1991)认为活性氧攻击叶绿素4吡咯环而导致卟 啉大环裂解, 加速了叶绿素的降解和含量降低。本 实验结果表明: 3种多胺不同程度地提高了叶片活 性氧清除酶(SOD、POD、CAT)的活性(图3), 从而抑 制了活性氧的生成速率, 延缓了叶绿素的降解。其 中外源 Spm对活性氧清除酶活性的作用效果最显 著。徐仰仓等(2001)对春小麦的研究表明: 外源Spm 既可直接作用于活性氧清除酶分子而提高活性, 也 可以诱导活性氧清除酶分子的合成。刘俊等(2006) 研究表明外施多胺通过提高玉米幼苗叶绿体结合态 多胺含量而增加叶片PSII光化学效率、净光合速率 和活性氧清除酶活性而缓解盐害, 其中Put的效果 最好。这与本实验花生结果略有差异, 可能是因为 植物种类的不同(Gill \& Tuteja, 2010)。

盐胁迫对质膜的伤害被认为是盐对植物的直接 原初伤害, 过剩的活性氧自由基引发或加剧膜脂过 氧化，造成细胞膜系统的损伤，导致细胞膜的透性

表2 盐胁迫下外源多胺对花生产量的影响(平均值土标准偏差)

Table 2 Effects of exogenous polyamines on the yield of Arachis hypogaea under salt stress (mean $\pm S D$ )

\begin{tabular}{|c|c|c|c|c|}
\hline $\begin{array}{l}\text { 处理 } \\
\text { Treatment }\end{array}$ & $\begin{array}{c}\text { 产量 } \\
\text { Pods mass per plant (g) }\end{array}$ & $\begin{array}{c}\text { 饱果数 } \\
\text { Full fruits number per plant }\end{array}$ & $\begin{array}{c}\text { 总果数 } \\
\text { Total pod number per plant }\end{array}$ & $\begin{array}{c}\text { 果针数 } \\
\text { Gynophores number per plant }\end{array}$ \\
\hline CK & $47.57 \pm 1.59^{\mathrm{a}}$ & $29.67 \pm 0.67^{\mathrm{a}}$ & $39.70 \pm 1.33^{\mathrm{a}}$ & $67.00 \pm 2.00^{\mathrm{a}}$ \\
\hline $\mathrm{NaCl}$ & $35.50 \pm 0.93^{c}$ & $16.21 \pm 0.33^{\mathrm{c}}$ & $30.67 \pm 2.67^{\mathrm{b}}$ & $40.67 \pm 1.48^{\mathrm{d}}$ \\
\hline $\mathrm{NaCl}+\mathrm{Put}$ & $39.17 \pm 0.87^{\mathrm{bc}}$ & $17.67 \pm 0.33^{\mathrm{bc}}$ & $31.00 \pm 0.67^{\mathrm{b}}$ & $43.00 \pm 2.67^{\mathrm{cd}}$ \\
\hline $\mathrm{NaCl}+\mathrm{Spd}$ & $40.25 \pm 1.13^{\mathrm{b}}$ & $21.00 \pm 1.24^{\mathrm{b}}$ & $31.67 \pm 0.48^{\mathrm{b}}$ & $45.00 \pm 1.67^{c}$ \\
\hline $\mathrm{NaCl}+\mathrm{Spm}$ & $41.50 \pm 1.32^{\mathrm{b}}$ & $20.67 \pm 0.67^{\mathrm{b}}$ & $39.33 \pm 1.24^{\mathrm{a}}$ & $57.00 \pm 3.33^{\mathrm{b}}$ \\
\hline
\end{tabular}

Put, 腐胺; Spd，亚精胺; Spm，精胺。同一列不同小写字母表示处理间差异显著 $(p<0.05)$ 。

Put, putrescine; Spd, spermidine; Spm, spermine. Different letters indicate significant differences among treatments $(p<0.05)$. 
增大(Larkindale \& Huang, 2004)。外源多胺的喷施使 得活性氧清除酶活性大幅提高, 从而有效地降低活 性氧, 减轻膜脂过氧化(图4)。

本实验结果表明：整体而言 Spm对花生盐胁迫 的缓解作用效果最好, 其原因可能在于多胺作用机 制的复杂性。多胺可以作用于植物的离子通道, 阻 断一种液泡膜上快速激活的离子通道, 从而阻断 $\mathrm{Na}^{+}$渗透到细胞质基质中(Bruggemann et al., 1998; Kusano et al., 2007a), 并且不同多胺的阻断强度可 能取决于多胺所带净电荷量, 即3 种常见多胺对离 子通道的阻断效果Spm > Spd $>>$ Put (Kusano et al., 2007b)。Yamaguchi等(2007)对缺失Spm拟南芥突变 体的研究则表明Spm可能通过控制钙渗透性通道调 节气孔关闭, 从而提高干旱胁迫下拟南芥的光合作 用。Jouve等(2004)、Ghosh等(2011)、Roychoudhury 等(2008)都发现植物耐盐性的提高与植物体内源 Spm含量的增加有关。内源多胺尤其是Spm提高植 物抗盐性可能与多胺调节质膜 $\mathrm{H}^{+}$-ATPase的活性有 关。Roy等(2005)发现耐盐植物根部的质膜处富含 Spd和Spm，而盐敏感型植物此处富含Put。

综上所述, 不同植物中多胺对盐胁迫的调节作 用机制可能在大田盆栽花生上也适用, 外源多胺的 施用, 尤其是 Spm, 减轻了盐胁迫对花生生长的危 害, 增加了盐胁迫下花生叶片叶绿素含量和抗氧化 酶活性，有利于光合作用的进行、干物质的积累。 在生产上, 花生的产量主要取决于花生植株的光合 作用能力及干物质积累速率。因此, 本研究为盐碱 地上花生的种植提供了参考。

基金项目 国家自然科学基金(31571581和 31571605)、国家科技支撑计划(2014BAD11B04)、 山东省农业重大应用技术创新课题、山东省自主创 新成果转化重大专项(2012ZHZXIA0418)、山东省自 然科学基金项目(ZR2011CQ042和ZR2015YL077)、 山东省农业科学院科技创新重点项目(2014CXZ066)、现代农业产业技术体系建设专项资金资助 (CARS-14)和山东省农业科学院青年科研基金 (2015YQN02)。

\section{参考文献}

Ndayiragije A, Lutts S (2007). Long term exogenous putrescine application improves grain yield of a salt-sensitive rice cultivar exposed to NaCl. Plant Soil, 291, 225-238.

Brüggemann LI, Pottosin II, Schönknecht G (1998). Cytoplasmic polyamines block the fast-activating vacuolar cation channel. The Plant Journal, 16, 101-105.

Diao FQ, Zhang WH, Liu YL (1997). Changes in composition and function of thylakoid membrane isolated from barley seedling leaves under salt stress. Plant Physiology, 23(2), 105-110. (in Chinese with English abstract) [ユ丰秋, 章 文华, 刘友良 (1997). 盐胁迫对大麦叶片类囊体膜脂组 成和功能的影响. 植物生理学报, 23(2), 105-110.]

Galston AW, Sawhney RK (1990). Polyamines in plant physiology. Plant Physiology, 94, 406-410.

Ghosh N, Adak MK, Ghosh PD, Gupta S, Sen Gupta DN, Mandal C (2011). Differential responses of two rice varieties to salt stress. Plant Biotechnology Reports, 5, 89-103.

Giannopolitis CN, Ries SK (1977). Superoxide dismutases II. Purification and quantitative relationship with watersoluble protein in seedling. Plant Physiology, 59, 315318.

Gill SS, Tuteja N (2010). Polyamines and abiotic stress tolerance in plants. Plant Signaling \& Behavior, 5, 26-33.

Jiang L, Shen ZY, Zhang ZL, Yan JQ (1993). The effect of polyamines on metabolism of active oxygen in detached leaves of Hordeum vulgare var. nudum Hook. f. Acta Phytophysiologica Sinica, 19, 367-371. (in Chinese with English abstract) [蒋琳, 沈曾佑, 张志良, 颜季琼 (1993). 多胺对裸大麦离体叶片活性氧代谢的影响. 植物生理 学报, 19, 367-371.]

Jouve L, Hoffmann L, Hausman JF (2004). Polyamine, carbohydrate, and proline content changes during salt stress exposure of aspen (Populus tremula L.): Involvement of oxidation and osmoregulation metabolism. Plant Biology, 6, 74-80.

Kusano T, Yamaguchi K, Berberich T, Takahashi Y (2007a). The polyamine spermine rescues Arabidopsis from salinity and drought stresses. Plant Signaling \& Behavior, 2, 251-252.

Kusano T, Yamaguchi K, Berberich T, Takahashi Y (2007b). Advances in polyamine research in 2007. Journal of Plant Research, 120, 345-350.

Krishnamurthy R, Bhagwat KA (1989). Polyamines as modulators of salt tolerance in rice cultivars. Plant Physiology, 91, 500-504.

Larkindale J, Huang B (2004). Changes of lipid composition and saturation level in leaves and roots for heat-stressed and heat-acclimated creeping bentgrass (Agrostis stolonifera). Environmental and Experimental Botany, 51, 57-67.

Li HS (2000). Principle and Technology of Plant Physiological and Biochemical Experiments. Higher Education Press, Beijing. 134-137. (in Chinese) [李合生 (2000). 植物生 理生化实验原理和技术. 高等教育出版社, 北京. 134-137.]

Li JG, Pu LJ, Zhu M, Zhang RS (2012). The present situation and hot issues in the salt-affected soil research. Acta Geographica Sinica, 67, 1233-1245. (in Chinese with 
English abstract) [ 李建国, 誉励杰, 朱明, 张润森 (2012). 土壤盐渍化研究现状及未来研究热点. 地理学 报, 67, 1233-1245.]

Liu J, Zhou YF, Zhang WH, Liu YL (2006). Effects of exogenous polyamines on chloroplast-bound polyamines content and photosynthesis of corn suffering salt stress. Acta Botanica Boreali-Occidentalia Sinica, 26, 254-258. (in Chinese with English abstract) [刘俊, 周一峰, 章文华, 刘友良 (2006). 外源多胺对盐胁迫下玉米叶绿体结合 态多胺水平和光合作用的影响. 西北植物学报, 26 , 254-258.]

Liu JH, Kitashiba H, Wang J, Ban Y, Moriguchi T (2007). Polyamines and their ability to provide environmental stress tolerance to plants. Plant Biotechnology, 24, 117126.

Liu K, Fu HH, Bei QX, Luan S (2000). Inward potassium channel in guard cells as a target for polyamine regulation of stomatal movements. Plant Physiology, 124, 13151325.

Maccarrone M, Baroni A, Finazzi-Agro A (1998). Natural polyamines inhibit soybean (Glycine max) lipoxygenase-1, but not the lipoxygenase-2 isozyme. Archives of Biochemistry and Biophysics, 356, 35-40.

Maslenkova LT, Zanev Y, Popova LP (1993). Adaptation to salinity as monitored by PSII oxygen evolving reactions in barley thylakoids. Plant Physiology, 142, 629-634.

Parida AK, Das AB (2005). Salt tolerance and salinity effects on plants: A review. Ecotoxicology and Environmental Safety, 60, 324-349.

Roy P, Niyogi K, SenGupta DN, Ghosh B (2005). Spermidine treatment to rice seedlings recovers salinity stress-induced damage of plasma membrane and PM-bound $\mathrm{H}^{+}$-ATPase in salt-tolerant and salt-sensitive rice cultivars. Plant Science, 168, 583-591.

Roychoudhury A, Basu S, Sarkar SN, Sengupta DN (2008). Comparative physiological and molecular responses of a common aromatic indica rice cultivar to high salinity with non-aromatic indica rice cultivars. Plant Cell Reports, 27, 1395-1410.

Roychoudhury A, Basu S, Sengupta DN (2011). Amelioration of salinity stress by exogenously applied spermidine or spermine in three varieties of indica rice differing in their level of salt tolerance. Journal of Plant Physiology, 168, 317-328.

Shu S, Yuan LY, Guo SR, Sun J, Yuan YH (2013). Effects of exogenous spermine on chlorophyll fluorescence, antioxidant system and ultrastructure of chloroplasts in Cucumis sativus L. under salt stress. Plant Physiology and Biochemistry, 63, 209-216.

Sudha G, Ravishankar GA (2003). Putrescine facilitated enhancement of capsaicin production in cell suspension cultures of Capsicum frutescens. Plant Physiology, 160,
339-346.

Sun C, Liu YL, Zhang WH (2002). Mechanism of the effect of polyamine on the activity of tonoplasts of barley roots under salt stress. Acta Botanica Sinica, 44, 1167-1172.

Wan SB (2009). Opportunities facing peanut industry in china and strategies for its science and technology development. Journal of Agricultural Science and Technology, 11(1), 7-12. (in Chinese with English abstract) [万书波 (2009). 我国花生产业面临的机遇与科技发展战略. 中国农业 科技导报, 11(1), 7-12.]

Wang XY, Ma CZ, Li XD (1999). Research on changes of polyamines during leaf senescence of different type peanut varieties. Chinese Journal of Oil Crop Sciences, 21(1), 31-34. (in Chinese with English abstract) [王晓云, 马池 珠, 李向东 (1999). 花生不同衰老型品种叶片衰老过程 中多胺变化规律的研究. 中国油料作物学报, 21(1), 31-34.]

Xu YC, Wang J, Liu H, Wang GX (2001). Promoting effect of exogenous spermine on anti-oxidative enzyme activity in wheat seedlings. Acta Phytophysiologica Sinica, 27, 349352. (in Chinese with English abstract) [徐仰仓, 王静, 刘 华, 王根轩 (2001). 外源精胺对小麦幼苗抗氧化酶活性 的促进作用. 植物生理学报, 27, 349-352.]

Yamaguchi K, Takahashi Y, Berberich T, Imai A, Takahashi T, Michael AJ, Kusano $\mathrm{T}$ (2007). A protective role for the polyamine spermine against drought stress in Arabidopsis. Biochemical and Biophysical Research Communications, 352, 486-490.

Shioi Y, Tatsumi Y, Shimokawa K (1991). Enzymatic degradation of chlorophyll in Chenopodium album. Plant Cell Physiology, 32, 87-93.

Zeng SX, Wang YR, Li MR (1997). Comparison of the changes of membrane protective system in rice seedlings during enhancement of chilling resistance by different stress pretreatment. Acta Botanica Sinica, 39, 308-314. (in Chinese with English abstract) [曾韶西, 王以柔, 李美如 (1997). 不同胁迫预处理提高水稻幼苗抗寒性期间膜保护系统 的变化比较. 植物学报, 39, 308-314.]

Zhang ZA, Chen ZY (2008). Plant Physiology Experiment Technology. Changchun University Press, Jilin. (in Chinese) [张治安, 陈展宇 (2008). 植物生理学实验技术.吉 林大学出版社, 长春.]

Zhao SJ, Xu CC, Zou Q, Meng QW (1994). Improvements of method for measurement of malondialdehyde in plant tissue. Plant Physiology Communications, 30(3), 207-210. (in Chinese) [赵世杰, 许长城, 邹琦, 孟庆伟 (1994). 植 物组织中丙二醛测定方法的改进. 植物生理学通讯, 30(3), 207-210.]

责任编委: 冯兆忠 责任编辑: 李 敏 